\title{
The Inverse Scattering Method Approach to the Quantum Shabat-Mikhailov Model
}

\author{
A. G. Izergin and V. E. Korepin \\ Leningrad Department of V. A. Steklov Mathematical Institute, \\ Fontanka 27, Leningrad 191011, USSR
}

\begin{abstract}
The Shabat-Mikhailov model is treated in the framework of the quantum inverse scattering method. The Baxter's $R$-matrix for the model is calculated.
\end{abstract}

\section{Introduction}

In this paper we consider the Shabat-Mikhailov model. This model was introduced in [1]. This model was investigated in detail for the first time in [2]. The solution of the equation of motion by means of the inverse scattering method was given in [3]. The $S$-matrix approach to the quantum version of the model was applied in [4], where the scattering matrix for the physical particles was calculated.

Here we consider the quantum version of the model, our approach being based on the quantum inverse scattering method [5]. This method is a generalization to the quantum case of the classical inverse scattering method proposed in [6].

The quantum inverse scattering method was successfully applied to the sineGordon model [7] which is somewhat similar to the model under consideration. The Hamiltonian structure of the model is of special interest for us. The angleaction variables in the framework of the classical inverse scattering method can be given in terms of scattering data [8]. One can easily express the scattering data by the monodromy matrix elements, the Poisson brackets of these elements being readily calculated by means of the classical $r$-matrix [9]. In Section 2 we list the main properties of the classical model and calculate the $r$-matrix.

In the quantum inverse scattering method, the Baxter's "quantum" $R$-matrix determining the commutation relations of the quantum monodromy matrix elements is important. The knowledge of these relations allows in principle the construction of all eigenfunctions of the Hamiltonian operator [5]. We propose a method of calculation of the $R$-matrix for the integrable field theory models based on the Yang-Baxter relations [10-14], the explicit form of the classical $r$-matrix, and the symmetry group of the models. For the classical version of the model considered, the corresponding symmetry group was introduced in [3]. 
In Sect. 3 we calculate the $R$-matrix for the Shabat-Mikhailov model. This $R$-matrix is the main result of the paper. Note that the Yang-Baxter relations are widely discussed in the literature, the solutions of these equations being of importance for the problems of quantum field theory and statistical physics (see $[5,14])$. In Sect. 4 the quantum monodromy matrix is considered. In Sect. 5 the further properties of the $R$-matrix are discussed. We construct an integrable spin model associated with the $R$-matrix. The connection between this model and the Shabat-Mikhailov model is the same as between the $X X Z$ and the sine-Gordon models.

It should be noted that the existence of the $R$-matrix means that the quantum version of the Shabat-Mikhailov model is also completely integrable. This model and the sine-Gordon one are the only integrable relativistic models containing one scalar field. The previous version of our results was published in [15].

\section{The Classical Model}

In this Section we represent the properties of the model in the form helpful for quantization. The Shabat-Mikhailov model is a relativistic model of one scalar field in two space-time dimensions. The Lagrangian and the equation of motion read as follows:

$$
\begin{array}{r}
\mathscr{L}=\gamma^{-1} \int d x\left[\frac{1}{2}\left(\partial_{\mu} u\right)^{2}-m^{2}\left(\exp (u)+\frac{1}{2} \exp (-2 u)-\frac{3}{2}\right)\right] ; \\
\square u+m^{2}(\exp (u)-\exp (-2 u))=0 ; \quad \square=\partial_{t}^{2}-\partial_{x}^{2} .
\end{array}
$$

This equation is valid if and only if the following operators $X(\lambda)$ and $M(\lambda)$ commute at any value of the spectral parameter $\lambda[3]$ :

$$
\begin{gathered}
{[X(\lambda), M(\lambda)]=0} \\
X(\lambda)=\partial_{x}+Q(\lambda) ; Q(\lambda)=Q_{1}(\lambda)+Q_{2}(\lambda)+Q_{3}(\lambda) ; \\
M(\lambda)=\partial_{t}+M_{1}(\lambda)+M_{2}(\lambda)+M_{3}(\lambda) .
\end{gathered}
$$

The matrices $Q_{i}, M_{k}$ are the $3 \times 3$-matrices, their nonzero elements being equal to:

$$
\begin{aligned}
& \left(Q_{1}\right)_{22}=-\left(Q_{1}\right)_{33}=-\frac{1}{2} \partial_{t} u \\
& \left(Q_{2}\right)_{23}=\frac{i}{2} m \lambda^{-1} \exp (-u) ;\left(Q_{2}\right)_{32}=-\frac{i}{2} m \lambda \exp (-u) \\
& \left(Q_{3}\right)_{12}=\left(Q_{3}\right)_{31}=\frac{i}{2} m \lambda^{-1} \exp \left(\frac{u}{2}\right) ;\left(Q_{3}\right)_{13}=\left(Q_{3}\right)_{21}=-\frac{i}{2} m \lambda \exp \left(\frac{u}{2}\right) \\
& \left(M_{1}\right)_{22}=-\left(M_{1}\right)_{33}=-\frac{1}{2} \partial_{x} u \\
& \left(M_{2}\right)_{23}=\frac{i}{2} m \lambda^{-1} \exp (-u) ;\left(M_{2}\right)_{32}=\frac{i}{2} m \lambda \exp (-u) ; \\
& \left(M_{3}\right)_{12}=\left(M_{3}\right)_{31}=\frac{i}{2} m \lambda^{-1} \exp \left(\frac{u}{2}\right) ;\left(M_{3}\right)_{13}=\left(M_{3}\right)_{21}=\frac{i}{2} m \lambda \exp \left(\frac{u}{2}\right)
\end{aligned}
$$

Note that operators $X, M$ naturally contain the corresponding operators for 
the Liouville equation. Namely the commutativity condition for the operators

$$
X^{(1)}=\partial_{x}+Q_{1}+Q_{2} ; M^{(1)}=\partial_{t}+M_{1}+M_{2}
$$

gives the equation $\square u-m^{2} \exp (-2 u)=0$ and the operators

$$
X^{(2)}=\partial_{x}+Q_{1}+Q_{3} ; M^{(2)}=\partial_{t}+M_{1}+M_{3}
$$

lead to the equation $\square u+m^{2} \exp (u)=0$.

Define now the monodromy matrix $T(x, y \mid \lambda)(x>y)$ :

$$
X(x \mid \lambda) T(x, y \mid \lambda)=0 ; T(y, y \mid \lambda)=I,
$$

the matrix $I$ being the unit $3 \times 3$-matrix. The specific form of the operator $X$ results in the existence of the symmetry group:

$$
\begin{gathered}
T^{*}(x, y \mid \lambda)=T\left(x, y \mid-\lambda^{*}\right) ; \\
q T\left(x, y \mid q_{-} \lambda\right) q^{-1}=T(x, y \mid \lambda) ; \\
p T^{T^{-1}}(x, y \mid-\lambda) p=T(x, y \mid \lambda) .
\end{gathered}
$$

The $3 \times 3$-matrices $p$ and $q$ are:

$$
p=\left(\begin{array}{ccc}
1 & 0 & 0 \\
0 & 0 & 1 \\
0 & 1 & 0
\end{array}\right) ; \mathrm{q}=\operatorname{diag}\left(1, q_{+}, q_{-}\right) ; q_{ \pm}=\exp \left( \pm \frac{2 \pi i}{3}\right) .
$$

This symmetry group will be essential for quantization. It follows from Eqs. (2), (7) that $\lim \operatorname{tr} T(x, y \mid \lambda)$ at $x \rightarrow \infty, y \rightarrow-\infty$ is time independent, hence $\ln \operatorname{tr} T(x, y \mid \lambda)$ is a generating functional for the conservation laws. The coefficients of $\ln \operatorname{tr}$ $T(x, y \mid \lambda)$ in a power expansion in $\lambda$ are the local conservation laws, the Hamiltonian being among them. To quantize the system, it is enough to find all the eigenfunctions of the trace of the monodromy matrix [5].

Let us now study the Hamiltonian aspects of the classical model. The basic Poisson brackets $(\mathrm{PB})$ are:

$$
\{\pi(x), u(y)\}=\delta(x-y) ; \pi(x)=\gamma^{-1} \partial_{t} u(x) .
$$

Our aim is to calculate the $\mathrm{PB}$ of the monodromy matrix elements. It is convenient to use the notations given below. The direct product of $3 \times 3$-matrices $A$ and $B$ we define as usual

$$
(A \otimes B)_{k \ell}^{i j}=A_{i j} B_{k \ell}(i, j ; k, \ell=1,2,3) .
$$

So we label matrix elements of $9 \times 9$-matrices by the "block" indices $i, j(i$ labels the block lines, $j$ the block columns) and by the internal indices $k, \ell$ ( $k$ labels the internal lines, $\ell$ the internal columns). Define also the PB of the direct product of the $3 \times 3$-matrices $A$ and $B$ :

$$
\{A \otimes, B\}_{k \ell}^{i j}=\left\{A_{i j}, B_{k \ell}\right\} .
$$

The right-hand side (r.h.s.) of Eq. (14) represents the $\mathrm{PB}$ of the two matrix elements of matrices $A, B$. The matrix product of two $9 \times 9$-matrices $C$ and $D$ in these 
notations can be written as:

$$
(C D)_{k \ell}^{i j}=C_{k n}^{i m} D_{n \ell}^{m j} .
$$

The summing up by the repeated indices is implied here.

To calculate the required $\mathrm{PB}$ we use the trick based on the classical $r$-matrix. If one succeeds in representing the $\mathrm{PB}$ of the matrix elements of $Q$ (Eq. (3)) as follows:

$$
\{Q(x \mid \lambda) \otimes, Q(y \mid \mu)\}=\gamma \delta(x-y)[r(\lambda, \mu), Q(x \mid \lambda) \otimes I+I \otimes Q(x \mid \mu)]
$$

then the $\mathrm{PB}$ of the monodromy matrix elements can be written as:

$$
\{T(x, y \mid \lambda) \otimes, T(x, y \mid \mu)\}=-\gamma[r(\lambda, \mu), T(x, y \mid \lambda) \otimes T(x, y \mid \mu)] .
$$

We give a particularly simple derivation of this formula in Appendix I. The square brackets at the r.h.s. of (16), (17) denote the commutator of two $9 \times 9$-matrices; $r$ is a numerical $9 \times 9$-matrix with elements depending on $\lambda$ and $\mu$. The existence of the $r$-matrix in (16) is not a priori evident. The direct calculation by means of (3), (4), (12) confirms, however, the validity of (16). We put the matrix $r$ into the form:

$$
r=\sum_{i, k, \ell, m=1}^{3} r_{\ell m}^{i k} e_{i k} \otimes e_{\ell m} .
$$

The $3 \times 3$-matrices $e_{i k}$ form the standard basis, their matrix elements being equal to:

$$
\left(e_{i k}\right)_{a b}=\delta_{i a} \delta_{k b}
$$

The matrix $r$ depends on $\lambda / \mu \equiv \exp (\beta)$ only: $r(\lambda, \mu)=r(\beta)$. The non-zero matrix elements are:

$$
\begin{aligned}
& r_{11}^{11}=(2-\exp (3 \beta)-\exp (-3 \beta)) g(\beta) ; \\
& r_{22}^{11}=r_{33}^{11}=r_{11}^{22}=r_{11}^{33}=-(2+\exp (3 \beta)+\exp (-3 \beta)) g(\beta) ; \\
& r_{33}^{22}=r_{22}^{33}=-2(\exp (3 \beta)+\exp (-3 \beta)) g(\beta) ; \\
& r_{21}^{12}=r_{13}^{31}=2(\exp (2 \beta)+\exp (-\beta)) g(\beta) ; \\
& r_{13}^{12}=r_{21}^{31}=2(\exp (2 \beta)-\exp (-\beta)) g(\beta) ; \\
& r_{31}^{13}=r_{12}^{21}=2(\exp (\beta)+\exp (-2 \beta)) g(\beta) ; \\
& r_{12}^{13}=r_{31}^{21}=2(\exp (-2 \beta)-\exp (\beta)) g(\beta) ; \\
& r_{32}^{23}=4 \exp (2 \beta) g(\beta) ; r_{23}^{32}=4 \exp (-2 \beta) g(\beta) ; \\
& g(\beta)=[4(\exp (3 \beta)-\exp (-3 \beta))]^{-1},
\end{aligned}
$$

The classical $r$-matrix is defined up to the addition of the arbitrary matrix proportional to the unit $9 \times 9$-matrix $E$ (see Eq. (16)).

The expression for the PB of the monodromy matrix elements is the main result of this Section. These formulae also determine the PB of the scattering data.

\section{The Quantum R-Matrix}

In this Section we consider the quantum version of the Shabat-Mikhailov model 
in the framework of the quantum inverse scattering method. The quantum model is given by the Lagrangian in (1) and the following commutation relations (CR) of the operators $u(x)$ and $\pi(x)=\gamma^{-1} \partial_{t} u(x)$ :

$$
[\pi(x), u(y)]=-i \delta(x-y)
$$

We will pay attention to the CR of the elements of the quantum monodromy matrix $\hat{T}(x, y \mid \lambda)$, this matrix being of importance also in the quantum inverse scattering method. For completely integrable quantum systems the following relation is valid $[11,5]$ :

$$
R(\lambda, \mu)(\hat{T}(x, y \mid \lambda) \otimes \hat{T}(x, y \mid \mu))=(\widehat{T}(x, y \mid \mu) \otimes \hat{T}(x, y \mid \lambda)) R(\lambda, \mu) .
$$

This relation is similar to (17) for the classical model. The elements of the $3 \times 3$ matrix $\hat{T}$ are quantum operators and $R(\lambda, \mu)$ is a numerical $9 \times 9$-matrix, depending also on $\gamma$.

Our purpose in this Section is to calculate the quantum $R$-matrix for the model. Note that (21) results in the following system of equations for $R(\lambda, \mu)$ (see Appendix 2):

$$
\begin{aligned}
(I \otimes R(\lambda, \mu))(R(\lambda, v) \otimes I)(I \otimes R(\mu, v)) \\
\quad=(R(\mu, v) \otimes I)(I \otimes R(\lambda, v))(R(\lambda, \mu) \otimes I) .
\end{aligned}
$$

The $27 \times 27$-matrix $(I \otimes R)$ is a direct product of the $3 \times 3$-unit matrix $I$ and $9 \times 9$ matrix $R$. Hence the 1.h.s. and r.h.s. of Eq. (22) contain the ordinary matrix product of three $27 \times 27$-matrices. Eq. (22) can be written in the explicit form as follows:

$$
R_{a_{3} c_{3}}^{a_{2} c_{1}}(\lambda, \mu) R_{c_{1} c_{2}}^{a_{1} b_{1}}(\lambda, v) R_{c_{3} b_{3}}^{c_{2} b_{2}}(\mu, v)=R_{a_{2} c_{2}}^{a_{1} c_{1}}(\mu, v) R_{a_{3} b_{3}}^{c_{2} c_{3}}(\lambda, v) R_{c_{3} b_{2}}^{c_{1} b_{1}}(\lambda, \mu) .
$$

These equations are the famous Yang-Baxter relations. Equations (22) and (23) are the basic ones for the calculation of the $R$-matrix.

A number of additional restrictions should be imposed on the $R$-matrix. The relativistic invariance implies that

$$
R(\lambda, \mu)=R(\lambda / \mu)
$$

The quasiclassical limit of $R$ is determined by the classical $r$-matrix. Comparing (17) and (21) one can obtain:

$$
\begin{aligned}
& R(\lambda / \mu) \underset{\gamma \rightarrow 0}{\simeq} R_{c}(\lambda / \mu) ; \\
& R_{c}(\lambda / \mu)=P(E-i \gamma r(\lambda / \mu)) .
\end{aligned}
$$

The $9 \times 9$ permutation matrix $P$ is defined by

$$
P_{k \ell}^{i j}=\delta_{i \ell} \delta_{j k} ; P^{2}=E
$$

and has the following properties

$$
P(C \otimes D) P=(D \otimes C) .
$$

Here $C, D$ are the numerical $3 \times 3$-matrices. Notice that the symmetries (Eqs. (8), (9), (10)) of the matrix $T$ result in the existence of the symmetry properties of the matrix $R_{c}$. We require these symmetries to be the same for the exact quantum 
matrix $R$ :

$$
\begin{gathered}
\left(q^{-1} \otimes q^{-1}\right) R(\lambda / \mu)(q \otimes q)=R(\lambda / \mu) ; \\
\left(I \otimes q^{-1}\right) R(\lambda / \mu)(q \otimes I)=R\left(q_{-} \lambda / \mu\right) ; \\
P R^{*}(\lambda / \mu) P=R\left(\mu^{*} / \lambda^{*}\right) ; \\
P R(\lambda / \mu) P=(p \otimes p) R^{T}(\lambda / \mu)(p \otimes p) .
\end{gathered}
$$

This requirement means that the classical symmetries survive after quantization. It is shown in Sect. 4 that the symmetries of $\hat{T}(x, y \mid \lambda)$ agree with Eqs. (27)-(30). Eq. (21) shows that the $R$-matrix is determined up to a scalar factor $f(\lambda, \mu)$. We choose this function $f$ implying the conditions:

$$
R(\lambda / \mu)=R^{-1}(\mu / \lambda) ; R(1)=E ; R_{22}^{22}(\lambda / \mu)=1 .
$$

Now we have written down all the equations which determine matrix $R$.

We succeeded in finding the $R$-matrix satisfying all these equations [15]. This matrix can be represented as the product of five $9 \times 9$-matrices:

$$
\begin{aligned}
R(\lambda / \mu ; \varphi) & =(I \otimes a(\lambda / \mu)) \mathrm{U}^{-1}(\varphi) D(\lambda / \mu ; \varphi) U(\varphi)\left(a^{-1}(\lambda / \mu) \otimes I\right) ; \\
\varphi & \equiv \gamma / 8 .
\end{aligned}
$$

We use the following notations. The elements of the diagonal matrix $D$ is equal to

$$
\begin{aligned}
D_{11}^{11} & =\cosh ((3 \beta / 2)+3 i \varphi) / \cosh ((3 \beta / 2)-3 i \varphi) ; \\
D_{33}^{11} & =D_{11}^{22}=D_{22}^{33} \\
& =-\sinh ((3 \beta / 2)+2 i \varphi) / \sinh ((3 \beta / 2)-2 i \varphi) ; \\
D_{22}^{11} & =D_{22}^{22}=D_{33}^{22}=D_{11}^{23}=D_{33}^{33}=1 ; \lambda / \mu=\exp (\beta) .
\end{aligned}
$$

The nonzero elements of the matrix $U(\varphi)$ are:

$$
\begin{aligned}
& U_{11}^{11}=U_{22}^{22}=U_{22}^{33}=U_{33}^{33}=-U_{23}^{32}=1 ; \\
& U_{22}^{11}=-U_{13}^{12}=U_{33}^{11}=U_{11}^{22}=U_{11}^{33}=\exp (i \varphi) ; \\
& \mathrm{U}_{21}^{12}=-U_{12}^{13}=-U_{31}^{13}=-U_{12}^{21}=U_{13}^{31}=\exp (-i \varphi) \\
& U_{32}^{23}=\exp (2 i \varphi) ; U_{33}^{22}=\exp (-2 i \varphi) ; \\
& U_{31}^{21}=2 \cos \varphi ; U_{21}^{31}=-2 i \sin \varphi .
\end{aligned}
$$

The diagonal $3 \times 3$-matrix $a(x)$ is:

$$
a(x)=\operatorname{diag}\left(1, x, x^{-1}\right) ; a(\lambda / \mu)=\left.a(x)\right|_{x=\lambda / \mu} .
$$

The nonzero matrix elements of the matrix $R$ are situated at the same places as the nonzero matrix elements of the matrix $U$.

The explicit expression for the matrix $R$ is our principal result.

Let us make now some remarks. It follows from the explicit expression of the $R$-matrix that it also possesses the following additional symmetries:

$$
\begin{gathered}
R^{T}=R ; \\
(a(x) \otimes a(x)) R(\lambda / \mu ; \varphi)=R(\lambda / \mu ; \varphi)(a(x) \otimes a(x)) ; x \in \mathbb{C} .
\end{gathered}
$$


One can show using this property that the following matrix $R_{z}(\lambda / \mu ; \varphi)$ is also the solution of the Yang-Baxter equations:

$$
R_{z}(\lambda / \mu ; \varphi)=\left(I \otimes a^{-1}\left((\lambda / \mu)^{z}\right)\right) R(\lambda / \mu ; \varphi)\left(a\left((\lambda / \mu)^{z}\right) \otimes I\right) .
$$

So we present the two-parameter solution of Eq. (22), the parameters being $z$ and $\varphi$. The matrix $R_{z}$ at $z=1$ is particularly simple:

$$
R_{1}(\lambda / \mu ; \varphi)=U^{-1}(\varphi) D(\lambda / \mu ; \varphi) U(\varphi) .
$$

This matrix is a meromorphic function of $(\lambda / \mu)^{3}$. The sine-Gordon $R$-matrix possesses the analogous properties (see Appendix 3). Equation (39) means that the eigenvectors of the matrix $R_{1}$ are $(\lambda / \mu)$-independent; the eight-vertex model $R$-matrix [11] has the same property. Note that in spite of the fact that our $R$-matrix is somewhat similar to the $R$-matrix [11], it is also has some new features. For example it can not be represented in the form

$$
R=\sum_{\alpha=1}^{9} W_{\alpha} I_{\alpha} \otimes I_{\alpha}
$$

at any choice of the basic $3 \times 3$-matrices $I_{\alpha}$.

\section{The Quantum Monodromy Matrix}

Here we consider the basic properties of the quantum monodromy matrix $\hat{T}(x, y \mid \lambda)$. The Hamiltonian, as in the classical case, is simply expressed in terms of the monodromy matrix. We define $\hat{T}(x, y \mid \lambda)$ as usual

$$
\hat{T}(x, y \mid \lambda)=\hat{L}_{N}(\lambda) \hat{L}_{N-1}(\lambda) \ldots \hat{L}_{1}(\lambda) .
$$

Here the interval $[x, y]$ is supposed to be divided into $N$ small segments of the length $\Delta(N \Delta=x-y)$. The operator $\hat{L}_{i}$ is the monodromy matrix for the $i$-th small segment. The CR of the matrix elements of the matrix $\hat{L}_{n}$ are given by the same formula (21) as the CR of the elements of $\hat{T}[5]$ :

$$
R(\lambda, \mu)\left(\hat{L}_{n}(\lambda) \otimes \hat{L}_{n}(\mu)\right)=\left(\hat{L}_{n}(\mu) \otimes \hat{L}_{n}(\lambda)\right) R(\lambda, \mu) .
$$

The matrix elements of the matrices $\hat{L}_{n}$ and $\hat{L}_{m}$ at $n \neq m$ commute. In the classical case the form of the matrix $L_{n}$ is quite clear. To construct the explicit form of the quantum operator, $\hat{L}_{n}$, is a dynamical problem. This problem is connected to the ultraviolet regularization problem, i.e. with the behavior of quantum local operators at small distances. In our case it would have been difficult to define the exact form of $\hat{L}_{n}$ before the calculation of the $R$-matrix (unlike the sine-Gordon model [7]). Now we are able to do this.

We will use Eq. (41) to find out the explicit form of the quantum operator $\hat{L}_{n}$. The matrix elements $\left(\hat{L}_{n}\right)_{i k} \equiv \ell_{i k}$ depend on $\lambda$ in the same way as the matrix elements of the potential $Q(\lambda)$ (Eqs. (3), (4)). The diagonal elements $\ell_{i i}=O(1)$, the nondiagonal elements $\ell_{i k}=O(\Delta)$. It follows from Eq. (41) that:

$$
\begin{aligned}
{\left[\ell_{11}, \ell_{i k}\right] } & =0 ;\left[\ell_{22}, \ell_{33}\right]=0 ; \\
\ell_{11}^{2} & =\ell_{22} \ell_{33} ; \ell_{11} \ell_{12}=\ell_{31} \ell_{22} \exp (-i \varphi) ; \ell_{11} \ell_{21}=\ell_{13} \ell_{22} \exp (-i \varphi) ;
\end{aligned}
$$




$$
\begin{aligned}
\ell_{11} \ell_{31} & =\ell_{33} \ell_{12} \exp (-i \varphi) ; \ell_{11} \ell_{13}=\ell_{33} \ell_{21} \exp (-i \varphi) ; \\
\ell_{a a} \ell_{1 b} & =\ell_{1 b} \ell_{a a} \exp \left\{(-1)^{a+1} 2 i \varphi\right\} \\
\ell_{a a} \ell_{b 1} & =\ell_{b 1} \ell_{a a} \exp \left\{(-1)^{a+1} 2 i \varphi\right\} ; \\
\ell_{a a} \ell_{23} & =\ell_{23} \ell_{a a} \exp \left\{(-1)^{a} 4 i \varphi\right\} \\
\ell_{a a} \ell_{32} & =\ell_{32} \ell_{a a} \exp \left\{(-1)^{a} 4 i \varphi\right\} \\
i, k & =1,2,3 ; a, b=2,3 .
\end{aligned}
$$

These CR can be realized as follows:

$$
\begin{gathered}
\ell_{11}=1 ; \ell_{12}=-\frac{i}{2} m \lambda^{-1} \Delta \exp \left(-\frac{i \varphi}{2}\right) \exp \left(\frac{u}{2}\right) \exp \left(\frac{p}{4}\right) ; \\
\ell_{13}=\frac{i}{2} m \lambda \Delta \exp \left(\frac{i \varphi}{2}\right) \exp \left(\frac{u}{2}\right) \exp \left(-\frac{p}{4}\right) ; \ell_{21}=-\lambda^{2} \ell_{12} ; \\
\ell_{22}=\exp \left(\frac{p}{2}\right) ; \ell_{23}=-\frac{i}{2} m \lambda^{-1} \Delta \exp (-u) ; \\
\ell_{31}=-\lambda^{-2} \ell_{13} ; \ell_{32}=-\lambda^{2} \ell_{23} ; \ell_{33}=\exp \left(-\frac{p}{2}\right) ; \\
u=\Delta^{-1} \int_{x_{n}}^{x_{n}+\Delta} u(y) d y ; p=\int_{x_{n}}^{x_{n}+\Delta} \partial_{t} u(y) d y ;[p, u]=-8 i \varphi .
\end{gathered}
$$

The operators $\ell_{i k}$ are defined up to terms $O\left(\Delta^{2}\right)$. So we have found the explicit form of the quantum monodromy matrix for a small segment $\Delta$. Using the fact that the quasiclassical limit of the operator $\exp (p)$ is equal to $1+\partial_{t} u\left(x_{n}\right) \Delta[7]$, one can easily prove that the quasiclassical limit of the operator $\hat{L}_{n}$ is correct.

Let us make now two remarks. The first one is that the matrix $R_{z}$ (Eq. (38)) "interlaces" the operators $\hat{L}_{z}$ :

$$
\begin{aligned}
& \hat{L}_{z}(\lambda)=a^{-1}\left(\lambda^{z}\right) \hat{L}(\lambda) a\left(\lambda^{z}\right) \\
& R_{z}(\lambda / \mu)\left(\widehat{L}_{z}(\lambda) \otimes \hat{L}_{z}(\mu)\right)=\left(\hat{L}_{z}(\mu) \otimes \hat{L}_{z}(\lambda)\right) R_{z}(\lambda / \mu) .
\end{aligned}
$$

The operator $\hat{L}_{1}$ corresponding to $R_{1}$ (Eq. (39)) is also a meromorphic function of $\lambda^{3}$. Secondly, the quantum operators $\hat{L}^{(1)}(\lambda), \hat{L}^{(2)}(\lambda)$, corresponding to the Liouville equations, can be constructed out of the same matrix elements of the operator $\hat{L}_{n}(\lambda)($ Eq. (43)) as in the classical case (5), (6). Our $R$-matrix (32) intertwines separately the operators $\hat{L}^{(1)}$ and the operators $\hat{L}^{(2)}$ in the sense of (41).

Now we return to the symmetries of $\hat{T}$. Direct calculations bring one to the following formulae

$$
\begin{gathered}
\hat{L}_{n}^{+}\left(-\lambda^{*}\right)=\widehat{L}_{n}(\lambda) ; \\
q \hat{L}_{n}\left(q_{-} \lambda\right) q^{-1}=\widehat{L}_{n}(\lambda) ; \\
p\left(\hat{L}_{n}^{-1}(-\lambda)\right)^{T} p=a\left(e^{-i \varphi}\right) \hat{L}_{n}\left(\lambda e^{2 i \varphi}\right) a^{-1}\left(e^{-i \varphi}\right), \\
p\left(\hat{L}_{n}^{T}(-\lambda)\right) p^{-1}=a\left(e^{i \varphi}\right) \widehat{L}_{n}\left(\lambda e^{-2 i \varphi}\right) a^{-1}\left(e^{i \varphi}\right) .
\end{gathered}
$$

The cross in (44) means the hermitian conjugation acting on the quantum operators and not on the matrix structure of the matrix $\hat{L}_{n}$. The matrix $a(x)$ is defined by (35). 
It follows from Eq. (40) that the monodromy matrix $\hat{T}$ possesses literally the same involutions as the matrix $\hat{L}_{n}$. The action of the symmetry group (44), (45), (46) turns into the classical one (8), (9), (10) in the quasiclassical limit $\varphi \rightarrow 0$. The symmetry properties of the $R$-matrix (Eqs. (27)-(30)) and of the matrix $T$ (Eqs. (44)(46)) agree with the Eq. (21) due to Eqs. (37), (24).

In this paper we will not discuss the properties of the quantum monodromy matrix in more detail, expecting to return in another paper to the construction of Bethe Ansatz, the physical vaccum and mass spectrum for the model.

\section{The Properties of the $\boldsymbol{R}$-Matrix}

In this Section we describe the lattice model associated with the $R$-matrix defined by (32) and construct the Zamolodchikov-Cherednik operators $A^{a}(\lambda)$ for this matrix.

First of all we describe the completely integrable lattice model, the construction of this model being similar to the construction of Baxter's eight-vertex model $[11,14]$. For this purpose we rewrite the "trilinear" relation (22) in the "bilinear" form similar to (41). Let us introduce nine $3 \times 3$-matrices $\tilde{L}_{a}^{b}$ (the indices $a, b=$ $1,2,3$ enumerate the matrices as a whole). We shall regard each individual matrix $\widetilde{L}_{a}^{b}$ as the "quantum operator". The matrices $\widetilde{L}_{a}^{b}$ are defined as follows:

$$
\left(\tilde{L}_{a}^{b}(\lambda)\right)_{k}^{i}=R_{a k}^{i b}(\lambda) .
$$

Here $i, k=1,2,3$ are matrix (quantum) indices of the matrix $\widetilde{L}_{a}^{b}$. Using these notations one can put Eq. (22) into the form similar to (41):

$$
R_{a_{3} c_{3}}^{a_{2} c_{2}}(\lambda / \mu) \tilde{L}_{c_{2}}^{b_{1}}(\lambda) \tilde{L}_{c_{3}}^{b_{2}}(\mu)=\tilde{L}_{a_{2}}^{c_{1}}(\mu) \tilde{L}_{a_{3}}^{c_{2}}(\lambda) R_{c_{2} b_{2}}^{c_{1} b_{1}}(\lambda / \mu) .
$$

Equation (48) shows that the operators $\widetilde{L}_{a}^{b}$ form in a natural way the $3 \times 3$-matrix $\tilde{L}$ which is similar to the matrix $\hat{L}_{n}$ from (40), (43). It should be noted that it was convenient for us to prove the validity of Eq. (22) in the form (48).

Equation (48) shows that the following model on the square $N \times M$ periodic two-dimensional lattice is integrable; the transfer-matrix $\tau$ of the model is defined as

$$
\tau(\lambda)=\sum_{a_{1} \ldots a_{N}}\left(\tilde{L}_{N}(\lambda)\right)_{a_{N}}^{a_{N-1}}\left(\tilde{L}_{N-1}(\lambda)\right)_{a_{N-1}}^{a_{N-2}} \ldots\left(\tilde{L}_{1}(\lambda)\right)_{a 1}^{a_{N}} .
$$

The main property of this transfer-matrix is

$$
[\tau(\lambda), \tau(\mu)]=0 .
$$

The partition function $Z$ is expressed as usual:

$$
Z=\operatorname{Sp} \tau^{M}
$$

(our notation for the transfer matrix differs from the notation in [14]). $\tau$ is an operator in the "quantum" space, in other words $\tau$ is the $3^{N} \times 3^{N}$-matrix.

This lattice model generates in a standard way $[16]$ the integrable spin model on the one-dimensional periodic lattice with $N$ sites. The Hamiltonian of the 
model reads:

$$
H=\left.4 \sinh 2 \psi \cosh 3 \psi\left(\tau^{-1} \frac{d \tau}{d \lambda}\right)\right|_{\lambda=1} ; \psi=i \varphi
$$

$H$ can be readily expressed through $d R(\lambda) / d \lambda$ at $\lambda=1$. This Hamiltonian is real and bounded below at the real $\psi$. To put it down in the explicit form we introduce eight "spin" matrices $I_{\alpha}^{n}$ at each site $(\alpha=1, \ldots, 8 ; n=1, \ldots, N)$, the spin $I^{n}$ acting nontrivially only at the $n$-th site:

$$
\begin{aligned}
& I_{1}=\left(2 e_{11}-e_{22}-e_{33}\right) / \sqrt{6} ; I_{2}=\left(e_{22}-e_{33}\right) / \sqrt{2} ; \\
& I_{3}=e_{12} ; I_{4}=e_{13} ; I_{5}=e_{21} ; I_{6}=e_{23} ; I_{7}=e_{31} ; \\
& I_{8}=e_{32} ;\left(e_{i k}\right)_{a b}=\delta_{i a} \delta_{k b} .
\end{aligned}
$$

The Hamiltonian (50) can be then rewritten as:

$$
H=\sum_{n=1}^{N} \sum_{\alpha, \beta=1}^{8} w_{\alpha \beta} I_{\alpha}^{n-1} I_{\beta}^{n}+h \sum_{n=1}^{N} I_{1}^{n}+\text { const, }
$$

the nonzero coefficients being equal to

$$
\begin{aligned}
h & =2 \sqrt{2}(2 \cosh 5 \psi-3 \cosh 3 \psi+\cosh \psi) / \sqrt{3} \\
w_{11} & =\cosh 5 \psi-3 \cosh 3 \psi-4 \cosh \psi ; \\
w_{22} & =-3(\cosh 5 \psi+\cosh 3 \psi) \\
w_{12} & =-w_{21}=-\sqrt{3}(\sinh 5 \psi+\sinh 3 \psi) ; \\
w_{35} & =w_{53}=w_{47}=w_{74}=-6 \cosh 3 \psi ; \\
w_{34} & =w_{57}=6 \exp (-2 \psi) \sinh 2 \psi ; \\
w_{43} & =w_{75}=-6 \exp (2 \psi) \sinh 2 \psi ; \\
w_{68} & =w_{86}=-6 \cosh \psi .
\end{aligned}
$$

The first term in the expression for $H$ is the interaction of spins at the neighbor sites. The second term represents the interaction of the spin $I_{1}$ with the fixed homogeneous magnetic field.

To conclude we will construct the operators $A^{a}(\lambda)$ for the Shabat-Mikhailov model. The definition of these operators reads

$$
A^{a}(\lambda) A^{b}(\mu)=R_{b d}^{a c}(\mu / \lambda) A^{c}(\mu) A^{d}(\lambda) .
$$

Using the direct product symbol one can rewrite this definition in the following form:

$$
(A(\lambda) \otimes A(\mu))=R(\mu / \lambda)(A(\mu) \otimes A(\lambda)) .
$$

It is shown in papers $[17,18]$ that under some assumptions Eq. (53) implies Eq. (22). We present the corresponding arguments in Appendix 2. The operators $A(\lambda)$ for the Baxter's model are constructed in [18]. The explicit form of the operators for the sine-Gordon model is given in Appendix 3.

In the Shabat-Mikhailov model the operators $A^{a}$ form the 3-component column, $(A \otimes A)$ being the 9-component column. We take the $\lambda$-dependence of 
$A(\lambda)$ in the following form:

$$
\begin{aligned}
& A_{1}(\lambda)=A_{1} ; \\
& A_{2}(\lambda)=a \lambda+b \lambda^{-2} ; \\
& A_{3}(\lambda)=c \lambda^{-1} .
\end{aligned}
$$

Equation (53) is then reduced to the following relations:

$$
\begin{aligned}
A_{1} a & =a A_{1} \exp (2 i \varphi) ; \quad A_{1} b=b A_{1} \exp (-2 i \varphi) ; \\
A_{1} c & =c A_{1} \exp (-2 i \varphi) ; \quad b c=c b \exp (4 i \varphi) ; \\
a c & =c a \exp (-4 i \varphi) ; \quad a b=b a \\
A_{1}^{2} & =(\exp (3 i \varphi)+\exp (i \varphi)) a c .
\end{aligned}
$$

These relations can be realized by means of operators acting on the scalar functions of one variable, $f(x)$ :

$$
\begin{aligned}
A_{1} f(x) & =m \exp \left(z_{1} x\right) f\left(x+a_{1}\right) \\
A_{2} f(x) & =\lambda \exp \left(z_{2} x\right) f\left(x+a_{2}\right)+\rho \lambda^{-2} \exp \left(-z_{2} x\right) f\left(x-a_{2}\right) ; \\
A_{3} f(x) & =\lambda^{-1} \exp \left(z_{3} x\right) f\left(x+a_{3}\right) \\
m^{2} & =(\exp (3 i \varphi)+\exp (i \varphi)) \exp \left(a_{2} z_{3}-a_{1} z_{1}\right) .
\end{aligned}
$$

Here $\rho$ is an arbitrary constant and the parameters $a_{i}, z_{i}$ satisfy the following equations:

$$
2 a_{1}=a_{2}+a_{3} ; \quad 2 z_{1}=z_{2}+z_{3} ; \quad z_{2} a_{3}-a_{2} z_{3}=4 i \varphi .
$$

The formula (54) gives the explicit realization of the operators $A^{a}$.

\section{Appendix 1}

The monodromy matrix $T(x, y \mid \lambda)$ depends on dynamical variables $u(x), \pi(x)$ through the potential $Q(x \mid \lambda)$ only. So one can express the Poisson brackets of the monodromy matrix elements as follows:

$$
\begin{gathered}
\left\{T_{i j}(\lambda), T_{k \ell}(\mu)\right\}=\int_{y}^{x} d z_{\lambda} \int_{y}^{x} d z_{\mu}\left(\delta T_{i j}(x, y \mid \lambda) / \delta Q_{a b}\left(z_{\lambda} \mid \lambda\right)\right) \\
\cdot\left(\delta T_{k \ell}(x, y \mid \mu) / \delta Q_{c d}\left(z_{\mu} \mid \mu\right)\right)\left\{Q_{a b}\left(z_{\lambda} \mid \lambda\right), Q_{c d}\left(z_{\mu} \mid \mu\right)\right\} .
\end{gathered}
$$

One can calculate the variational derivative of $T(\lambda)$ with respect to $Q(\lambda)$ by means of the perturbation theory:

$$
\delta T(x, y \mid \lambda)=-\int_{y}^{x} T(x, z \mid \lambda) \delta Q(z \mid \lambda) T(z, y \mid \lambda) d z .
$$

Now it is possible to rewrite (A1) by means of (A2) and (13), (14), (15) in the elegant form:

$$
\begin{aligned}
& \{T(x, y \mid \lambda) \otimes \underset{,}{T} T(x, y \mid \mu)\}=\int_{y}^{x} d z_{\lambda} \int_{\nu}^{x} d z_{\mu}\left(T\left(x, z_{\lambda} \mid \lambda\right) \otimes T\left(x, z_{\mu} \mid \mu\right)\right) \\
& \cdot\left\{Q\left(z_{\lambda} \mid \lambda\right) \otimes \underset{,}{Q} Q\left(z_{\mu} \mid \mu\right)\right\}\left(T\left(z_{\lambda}, y \mid \lambda\right) \otimes T\left(z_{\mu}, y \mid \mu\right)\right) .
\end{aligned}
$$


Using (16)

$$
\left\{Q\left(z_{\lambda} \mid \lambda\right) \otimes \ni Q\left(z_{\mu} \mid \mu\right)\right\}=\gamma \delta\left(z_{\lambda}-z_{\mu}\right)\left[r, Q\left(z_{\lambda} \mid \lambda\right) \otimes I+I \otimes Q\left(z_{\lambda} \mid \mu\right)\right]
$$

one obtains

$$
\begin{aligned}
\{T(x, y \mid \lambda) \otimes \underset{,}{ } T(x, y \mid \mu)\}= & \gamma \int_{y}^{x} d z(T(x, z \mid \lambda) \otimes T(x, z \mid \mu)) \\
& \cdot[r(\lambda, \mu), Q(z \mid \lambda) \otimes I+I \otimes Q(z \mid \mu)](T(z, y \mid \lambda) \otimes T(z, y \mid \mu)) .
\end{aligned}
$$

To transform this expression we write (7) in the form:

$$
\begin{aligned}
& \partial_{x} T(x, y \mid \lambda)=-Q(x \mid \lambda) T(x, y \mid \lambda) \\
& \partial_{y} T(x, y \mid y)=T(x, y \mid \lambda) Q(y \mid \lambda) ; T(y, y \mid \lambda)=I
\end{aligned}
$$

Excluding the potential $Q(z \mid \lambda)$ in (A5) by means of (A6) one gets:

$$
\begin{gathered}
\{T(x, y \mid \lambda) \otimes \underset{,}{T} T(x, y \mid \mu)\}=-\gamma \int_{y}^{x} d z \frac{d}{d z}((T(x, z \mid \lambda) \otimes T(x, z \mid \mu)) \\
\cdot r(\lambda, \mu)(T(z, y \mid \lambda) \otimes T(z, y \mid \mu))) .
\end{gathered}
$$

Calculating the integral one comes to Eq. (17):

$$
\{T(x, y \mid \lambda) \otimes T(x, y \mid \mu)\}=-\gamma[r(\lambda, \mu), T(x, y \mid \lambda) \otimes T(x, y \mid \mu)]
$$

This way of evaluating the Poisson brackets is rather similar to the standard calculations by means of the "fourth power" identities [19].

\section{Appendix 2}

Let us write the definition (53) of the operators $A(\lambda)$ :

$$
(A(\mu) \otimes A(\lambda))=R(\lambda, \mu)(A(\lambda) \otimes A(\mu)) .
$$

Here $A^{\alpha}(\lambda)$ is the operator column. Consider the direct product of three such columns: $A(v) \otimes A(\mu) \otimes A(\lambda)$. One can transform this expression to the form $A(\lambda) \otimes A(\mu) \otimes A(v)$ by means of Eq. (A8) in two different ways:

$$
\begin{aligned}
& (A(v) \otimes A(\mu) \otimes A(\lambda)) \\
& \quad=(R(\mu, v) \otimes I)(I \otimes R(\lambda, v))(R(\lambda, \mu) \otimes I)(A(\lambda) \otimes A(\mu) \otimes A(v)) \\
& \quad=(I \otimes R(\lambda, \mu))(R(\lambda, v) \otimes I)(I \otimes R(\mu, v))(A(\lambda) \otimes A(\mu) \otimes A(v)) .
\end{aligned}
$$

Equation (22) is a consequence of (A9) provided that all the 27 components of the column $A \otimes A \otimes A$ are linearly independent. It should be noted that our operators $A^{a}(\lambda)$ from (54) are not of this type. So we had to prove the validity of Eq. (22) by means of direct calculations.

Another way to obtain Eq. (22) is to use (21) to transform the expression $T(\lambda) \otimes T(\mu) \otimes T(v)$ to the form $T(v) \otimes T(\mu) \otimes T(\lambda)$ in two different ways. 


\section{Appendix 3}

The sine-Gordon $R$-matrix can be represented in the form

$$
R(\alpha ; \gamma)=U^{-1} D(\alpha ; \gamma) U
$$

Here

$$
\begin{gathered}
D_{11}^{11}=D_{22}^{22}=1 ; \quad D_{22}^{11}=\cosh \left(\frac{\alpha-i \gamma}{2}\right) / \cosh \left(\frac{\alpha+i \gamma}{2}\right) ; \\
D_{11}^{22}=-\sinh \left(\frac{\alpha-i \gamma}{2}\right) / \sinh \left(\frac{\alpha+i \gamma}{2}\right) ; \\
U_{11}^{11}=U_{22}^{11}=-U_{11}^{22}=U_{22}^{22}=U_{21}^{12}=U_{12}^{21}=1 .
\end{gathered}
$$

This $R$-matrix generates the one-parametric family of solutions of Eq. (22):

$$
\begin{gathered}
R_{z}(\alpha ; \gamma)=\left(I \otimes a_{s}^{-1}\left(e^{z \alpha}\right)\right) R(\alpha ; \gamma)\left(\alpha_{s}\left(e^{z \alpha}\right) \otimes I\right) ; \\
a_{s}(x)=\operatorname{diag}\left(x, x^{-1}\right) .
\end{gathered}
$$

We will put down also the operators $A^{a}(\alpha)(a=1,2)$ satisfying Eq. (53) for the sine-Gordon $R$-matrix. Take the $\alpha$-dependence of the operators $A$ in the form:

$$
\begin{aligned}
& A^{1}(\alpha)=\exp (2 \alpha) a_{2}+a_{0}+\exp (-2 \alpha) a_{-2} ; \\
& A^{2}(\alpha)=\exp (\alpha) b_{1}+\exp (-\alpha) b_{-1} .
\end{aligned}
$$

The operators $a, b$ can be realized as follows:

$$
\begin{aligned}
& a_{-2} f(x)=\rho_{-2} \exp \left(z_{-2} x\right) f\left(x+k_{-2}\right) ; \\
& b_{-1} f(x)=\rho_{-1} \exp \left(z_{-1} x\right) f\left(x+k_{-1}\right) ; \\
& a_{2}=\rho_{2} a_{-2}^{-1} ; \quad a_{0}=\rho_{1} a_{-2}+\rho_{1}^{-1} a_{2} ; \quad b_{1}=\rho_{1} b_{-1} ; \\
& k_{-1} z_{-2}-k_{-2} z_{-1}=i \gamma .
\end{aligned}
$$

Here $\rho_{i}$ are the arbitrary constants.

Acknowledgements. We thank L. D. Faddeev for his interest in our work and useful discussions. We are thankful to A. V. Mikhailov who drew our attention to the problem. We appreciate discussions with L. A. Takhtadjan, P. P. Kulish, A. G. Reyman, V. O. Tarasov.

\section{References}

1. Dodd, R. K., Bullough, R. K. : Proc. R. Soc. Lond. A352, 481 (1977)

2. Zhiber, A. V., Shabat, A. B. : Dokl. Akad. Nauk SSSR 247, 1103 (1979)

3. Mikhailov, A. V. : Pis'ma Zh. Eksp. Teor. Fiz. 30, 443 (1979); preprint ITEF-44. Moscow (1980)

4. Arinshtein, A. E., Fateyev, V. A., Zamolodchikov, A. B.: Phys. Lett. 87B, 389 (1979)

5. Faddeev, L. D.: LOMI-preprint P-2-79. Leningrad (1979)

6. Gardner, C. S., Green, J. M., Kruskal, M. D., Miura, R. M. : Phys. Rev. Lett. 19, 1095-1097 (1967)

7. Faddeev, L. D., Sklyanin, E. K., Takhtadjan, L. A. : Teor. Mat. Fiz. 40, 194 (1979)

8. Faddeev, L. D., Zakharov, V. E. : Funk. Anal. Jego Prilozh. 5, 18 (1971)

9. Sklyanin, E. K. : LOMI-preprint E-3-1979. Leningrad (1979)

10. Yang, C. N. : Phys. Rev. Lett, 19, 1312-1315 (1967) 
11. Baxter, R. J. : Ann. Phys. (N Y). 70, 193-228 (1972); 70, 323-327 (1972); 76, 25-47 (1973); 76, 48-71 (1973)

12. Zamolodchikov, A. B. : Commun. Math. Phys. 55, 183 (1977)

13. Karowski, M., Thun, H. J., Truong, T. T., Weisz, P. H. : Phys. Lett. 67B, 321-322 (1977)

14. Faddeev, L. D., Takhtadjan, L. A. : Usp. Mat. Nauk. 34, 13-63 (1979)

15. Izergin, A. G., Korepin, V. E.: LOMI-preprint E-3-80. Leningrad (1980)

16. Sutherland, B.: J. Math. Phys. (NY), 11, 3183-3186 (1970)

17. Zamolodchikov, A. B. : Pis'ma Zh. Exp. Teor. Fiz. 25, 499-502 (1977)

18. Cherednik, I. V. Dokl. Akad. Nauk SSSR 249, 1095 (1979)

19. Zakharov, V. E., Manakov, S. V., Novikov, S. P., Pitajevsky, L. P. :The theory of solitons. Moscow: Nauk 1980

Communicated by Ya. G. Sinai

Received July 14, 1980 